\title{
Occult Prostate Cancer: Condylar Mandibular Metastasis Like Unique Manifestation. Rare case report, First Described in Italian Literature
} MG Cristofaro ${ }^{*}$, Giudice $A^{1}$, Colangeli $W^{1}$, Giofrè $E^{1}$, Riccelli $U^{1}$ and M Giudice ${ }^{1,2}$

${ }^{1}$ Department of Oral and Maxillofacial Surgery, University Magna Graecia Catanzaro, Italy

${ }^{2}$ Professor and Chairman, Department of Oral and Maxillofacial Surgery, University Magna Graecia Catanzaro, Italy

\begin{abstract}
Prostate cancer is the most frequently diagnosed cancer in men and the second leading cause of cancer death among men in the United States. The most common site of prostate cancer metastasis is the bone, with skeletal metastases identified at autopsy in up to $90 \%$ of patients dying from prostate cancer. The route of metastasis to bone is thought the prostatic venous plexus draining with the vertebral veins. In this report jaw bones metastases occur before the patient has been diagnosed a primary tumour; in a smaller rate their diagnoses coincides with the diagnosis of the primary tumour. Data reported in literature a low incidence of jaw bones metastases; they are even less recurrent in mandibular condyles owing to their low red bone marrow (hematopoietic active) content in adulthood. Just in a few exceptional cases, like this case, bone metastases is the first clinical evidence of an occult or initial cancer, a site occurs above all in prostate, bladder and lung cancer. Our case are exceptional because the mandibular condyle metastasis was the first clinical sign of an occult primary prostate carcinoma, whose early diagnosis made the treatment of both (primary tumour and sigle metastasis) more effective.
\end{abstract}

Keywords: Prostate cancer; Mandibular condyle metastasis; Jaw metastasis

\section{Introduction}

Prostate cancer (PCa) is the most frequently diagnosed cancer in men and the second leading cause of cancer death among men in the United States. The most common site of PCa metastasis is the bone, with skeletal metastases identified at autopsy in up to $90 \%$ of patients dying from PCa. Skeletal metastases are a significant cause of morbidity and mortality and overall greatly affect the quality of life of prostate cancer patients. Prostate cancer most commonly metastasizes not only to the bones but also to lymph nodes, and may invade rectum, bladder and lower ureters after local progression. The route of metastasis to bone is the draining of prostatic venous plexus into vertebral veins $[1,2]$.

However, bone metastasis in prostate cancer is underestimated since antiblastic treatments, which extend our patients' survival, further improve the chances for the recurrence of lesions.

The mainly are metastases due to carcinomas, which are defined osteotrope or osteophile due to their specific bone affinity. Among them, the most recurrent are: breast carcinoma (bone metastases in $78 \%$ of cases), prostatic adenocarcinoma (bone metastases in $54-73 \%$ of cases), lung carcinoma (bone metastases in 30\% of cases), thyroid carcinoma (bone metastases in $30-40 \%$ of cases), hypernephroma (bone metastases in $30 \%$ of cases), adrenal carcinomas and hepatocellular carcinoma (HCC) [3] in a smaller rate.

The physiochemical properties of bone, and signaling molecules including specific chemokines and their receptors, are distinct in nature and function, yet play intricate and significant roles in prostate cancer bone metastasis.

\section{Case Report}

A.S., aged 60, was referred to the Department of Oral and Maxillo Facial Surgery in October 2006 for swelling in left parotid region due to $2 \times 3 \mathrm{~cm}$, associated with pain. There were any satellite lymphoadenopathies and no disorders involving the $5^{\text {th }}$ and $7^{\text {th }}$ pair of left cranial nerves.

He underwent an Orthopantomografy (OPT), neck US (that highlighted a few reactive parotid lymph nodes large almost $16 \mathrm{~mm}$ ) and a Computerized Tomography (CT) that showed an osteolytic lesion in the left mandibular condyle and its pathological fracture (Figure 1). The functional assessment of TMJ joints proved an increase slight translation in the right condyle as against the left condyle one.

The bioptic examination of the condyle lesion tell us that it was a metastasis from an occult prostatic adenocarcinoma with a cribriform and micropappillary pattern (Figure 2,3). The patient underwent to an urologist. From the prostate US we find: prostate size was 4.7 x $4.5 \times 4.5$ $\mathrm{cm}$ with a $1 \mathrm{~cm}$ lesion in the left side of central gland. No other lesion in bladder e seminal vesicles.

The histological examination was performed by a prostate needle aspiration biopsy under ultrasound guidance on a poorly vascularised extra capsular $1.1 \mathrm{~cm}$-mass. It was hypoechogenic, and showed a conventional acinar adenocarcinoma of the prostate on the istological examination, whose overall Gleason scores were 7 with Gleason pattern 5 (Gleason score modified according to IUSP 2005) as well a perineural invasion (Figure 4). The patient underwent also to a Technetium99skeletal scintigraphy which highlighted a strong pathological increase in the uptake of the radioactive tracer only on the left mandibular

*Corresponding author: Cristofaro MG, Department of Oral and Maxillofacia Surgery, University Magna Graecia Catanzaro, Italy, E-mail: cristofaro@unicz.it

Received November 22, 2011; Accepted December 03, 2011; Published December 05, 2011

Citation: Cristofaro MG, Giudice A, Colangeli W, Giofrè E, Riccelli U, et al. (2011) Occult Prostate Cancer: Condylar Mandibular Metastasis Like Unique Manifestation. Rare case report, First Described in Italian Literature. J Cancer Sci Ther S1. doi:10.4172/1948-5956.S1-009

Copyright: ( 2011 Cristofaro MG, et al. This is an open-access article distributed under the terms of the Creative Commons Attribution License, which permits unrestricted use, distribution, and reproduction in any medium, provided the original author and source are credited. 
Citation: Cristofaro MG, Giudice A, Colangeli W, Giofrè E, Riccelli U, et al. (2011) Occult Prostate Cancer: Condylar Mandibular Metastasis Like Unique Manifestation. Rare case report, First Described in Italian Literature. J Cancer Sci Ther S1. doi:10.4172/1948-5956.S1-009

condyle and part of the ascending ramus of mandible (Figure 5). After this examination we planned a left subtotal hemimandibulectomy followed by a reconstruction with a titanium replacement plate with no microvascularized free flap for his peripheral vascular disease led us not to use free microvascularized flaps (Figure 6,7). The postoperative course was satisfactory and without any motor or sensory deficit. Clinical follow-ups were carried out after 1, 3, 6 and 12 months; 6

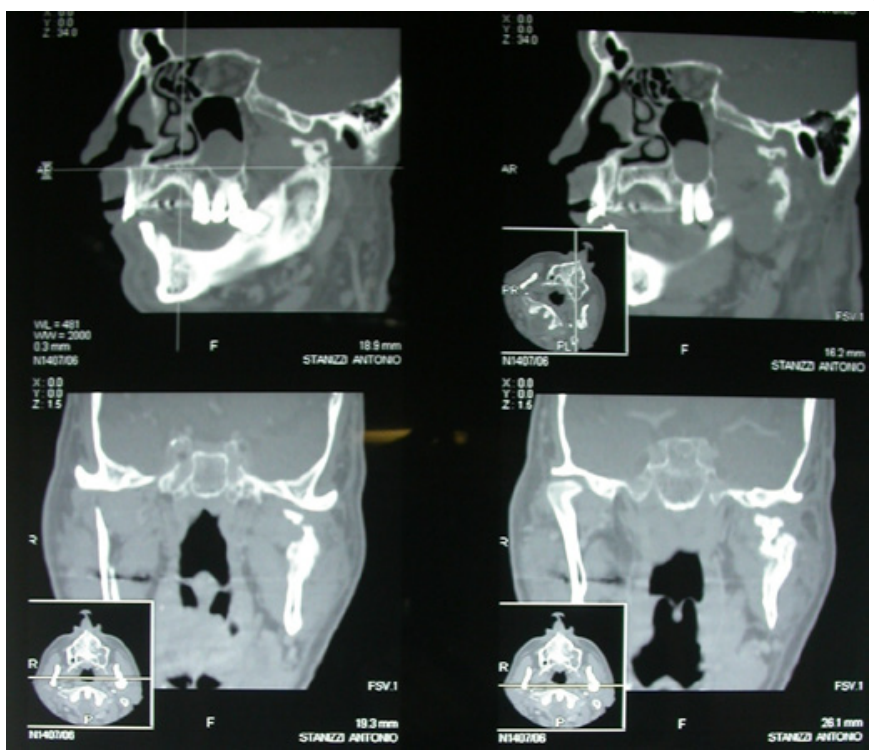

Figure 1: Preoperative CT revealing an osteolytic lesion with mandibularcondyle pathologic fracture.

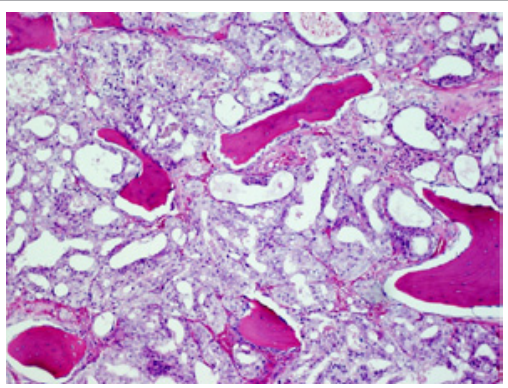

Figure 2: Microscopic appearance of prostatic carcinoma. Adenocarcinoma composed of small glands, the individual glands having a irregular round configuration and cribriform pattern.

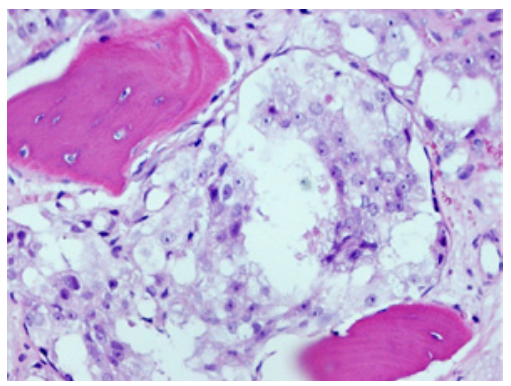

Figure 3: Both of these architectural patterns are accompanied by cytological abnormalities in the form of nuclear enlargement, hypercromasia, and prominent nucleoli.

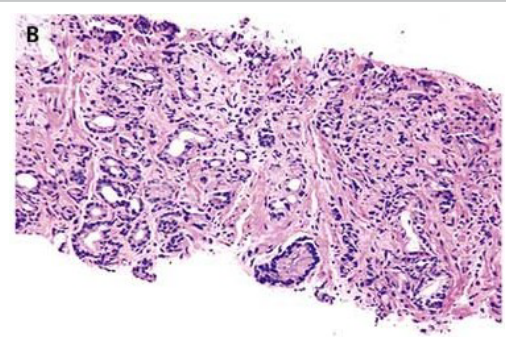

Figure 4: Microscopic appearance of prostatic carcinoma. Gleason Score 7 with pattern 5 .

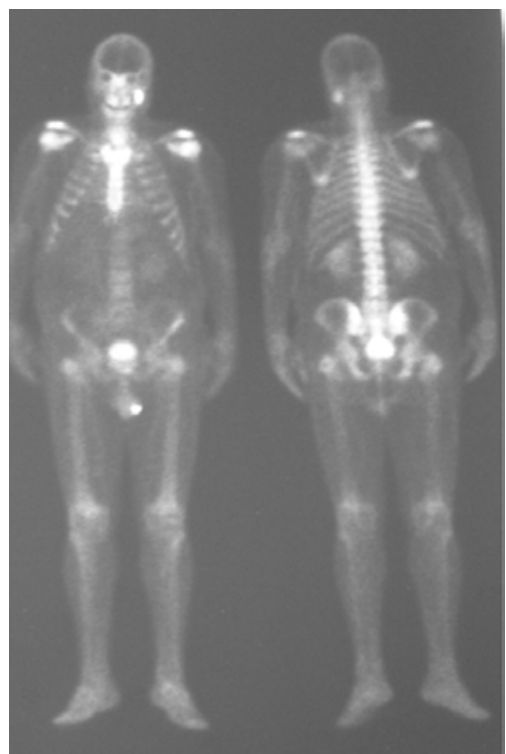

Figure 5: Total body scintigraphy that shows a strong pathological incensement of the tracer on the condyle mandibular left and part of mandible ascending ramus.

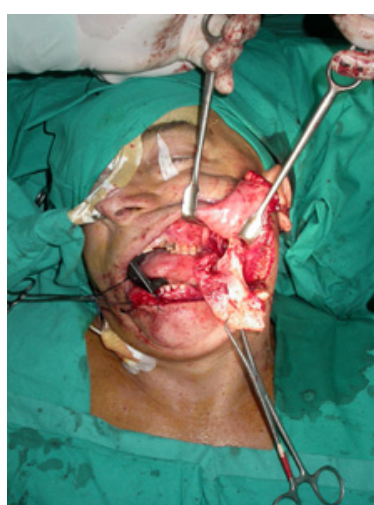

Figure 6: Intraoperative views

months later the patient underwent a skull CT and 12 months later a OPT (Figure 8) and skeletal scintigraphy (Figure 9); the final follow -up was performed two years after the surgery and it did not show any recurrence. The patient also underwent chemotherapy immediately in order to treat his primary tumour and radiotherapy of his mandibular condyle metastasis during the preoperative stage. 
Citation: Cristofaro MG, Giudice A, Colangeli W, Giofrè E, Riccelli U, et al. (2011) Occult Prostate Cancer: Condylar Mandibular Metastasis Like Unique Manifestation. Rare case report, First Described in Italian Literature. J Cancer Sci Ther S1. doi:10.4172/1948-5956.S1-009

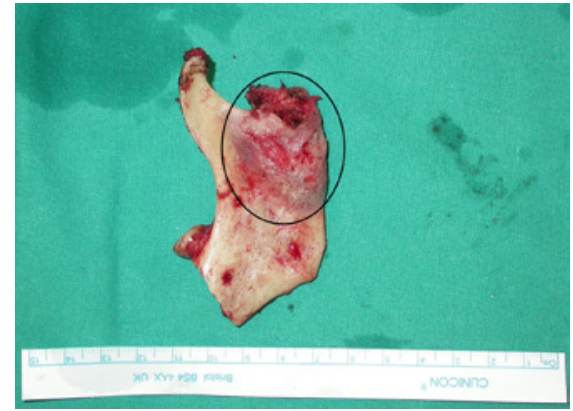

Figure 7: Intraoperative views.

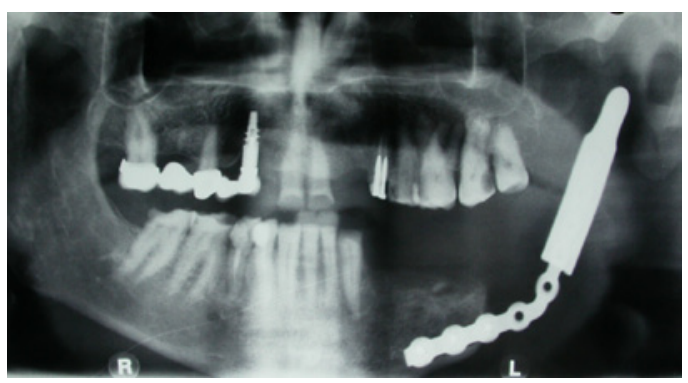

Figure 8: XR control: OPT after 12 months since surgery.

\section{Discussion}

The literature suggests that jaws are not a common site of prostatic metastasis ( 1 per- cent of all metastatic bone lesions. This low rate is linked to low active bone marrow content in jaw bones of adult patients [4-6]; just 40 cases had been reported in international literature [7].

In the majority of patients that present an oral metastasis, the primary tumor has generally been well diagnosed and treated. However in a small number of patients the oral metastasis represents the initial finding which ultimately leads to the detection of a hidden malignant lesion. The frequency of metastatic oral lesions is low and is one of the reasons why it can present as a difficult diagnostic challenge [8]. Actually, it is rather difficult to diagnose metastases in jaw bones and namely in the mandible: and indeed, they often are diagnosed late [9].

The spread out of metastases may occur through blood or the lymphatic system as well as owing to contiguity. In this case (in the maxilla), cancer cells alter the balance between osteoclast/osteoblast activity; in most cases osteoclast activity can be observed by $\mathrm{x}$-ray studies, showing the presence of an osteolytic area. More rarely we can notice an increase in osteoblast activity leading to a marked new bone formation; but these aspects are common in prostate, lung and bladder cancer. The gardening of the bone, which looks dense or slightly porous, could be consistent with a simple osteosclerosis. Their fast development causes some pathologic fractures, as in our report.

In this report jaw bones metastases occur before the patient has been

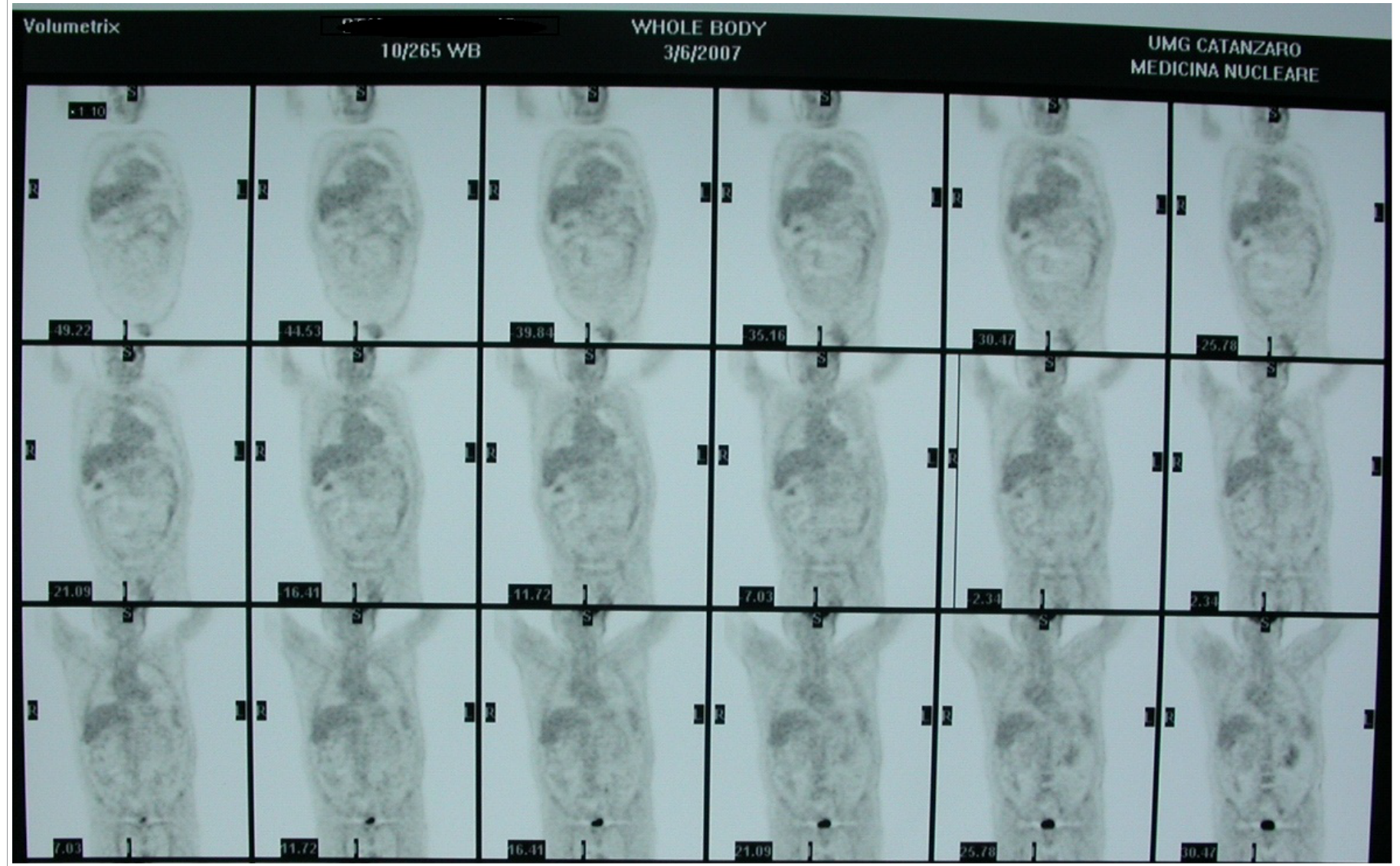

Figure 9: Total body scintigraphy after 12 months since surgery. 
diagnosed a primary tumour; in a smaller rate diagnosis coincides with the primary tumour. Just in a few exceptional cases bone metastases is the first clinical evidence of an occult or initial cancer, as it occurs above all in prostate, bladder and lung cancer $[10,11]$.

In a review of the literature, Hirshberg et al. [6] also reported that the breast and lung were the most common primary sites in women and men, respectively [12].

At the metastatic bone site, growth of PCa promotes localized bone remodeling that results in primarily osteosclerotic lesions (also known as osteoblastic lesions characterized by increased production of mineralized bone matrix resulting in increased bone mineral density) with underlying osteolytic lesions (i.e., osteoclast mediated resorption of mineralized bone matrix resulting in low bone mineral density; ). Although mechanisms contributing to the osteopenic component of PCa-mediated bone lesions have been elucidated, the mechanisms responsible for the osteoblastic component of PCa bone lesions are not well defined, although a variety of factors such as bone morphogenetic proteins (BMP), endothlin-1 (ET-1), vascular endothelial growth factor (VEGF) [9] have been implicated as contributing to osteoblastic metastasis. Understanding the mechanisms that promote PCa-induced osteosclerosis may help identify targets to diminish the progression of these lesions [13].

Strumental researches are important for diagnosis: OPT is the first examination that a patient had to do; it can reveal the presence of one or more radiopaque or mixed areas in jaw bones, in case of metastases deriving from prostate cancer, or lower rate of radiolucent areas. CT scan shows bone better team and pathological fracture as in our cases; MRI of jaw bones gave further details on the extent and infiltration of soft tissues; skeletal scintigraphy is an important test to asses the presence of metastases in other parts of the skeleton; biopsy of the lesion provided us the diagnosis.

The critical element to make diagnosis is provided by biopsy of specimens taken from a lesion. This Diagnosis was rather difficult beacause we dealt with a bone metastasis deriving from an occult primary carcinoma and the site of its recurrence was almost rare [14].

Treating these metastases is not easy since they are often diagnosed late and frequently we deal with patients suffering from any advanced - stage cancer, in which surgery would not improve the quality of their lives.

Radical surgery is indicated in single metastasis; it must be coupled with chemo-radiotherapy of the metastatic region. About $10 \%$ of precociously diagnosed cases shows a 4-year survival rate.

\section{Conclusion}

Data reported in literature highlight a low incidence of jaw bones metastases especially from prostatic cancer; owing to their low red bone marrow (hematopoietic active) content in adulthood.

Jaw bone metastasis and namely mandibular condyle metastasis, is endorsed by the hypothesis suggested by Batson [2], who identified a direct way for neoplastic cells from prostatic veins to the internal vertebral venous plexus, which has no valves, and reach the cranial bones, avoiding systemic circulation. The review of the literature shows the rarity of the mandibular condyle metastases that becomes exceptional, as in the case described by the authors, in which bone metastases is the first clinical evidence of an occult prostate cancer, which indicates that early diagnosis made more effective treatment (primitive and metastases lesion).

\section{References}

1. Kaufmann MG, Perren A, Grätz KW, Eyrich GK (2005) Condylar metastasis. Review of the literature and report of a case. Mund Kiefer Gesichtschir 9: 336340.

2. Batson OV (1940) The function of the vertebral veins and their role in the spread of metastasis. Ann Surg 112:138-149.

3. Kamatani T, Tatemoto Y, Tateishi Y, Yamamoto T (2008) Isolated metastasis from hepatocellular carcinoma to the mandibular condyle with no evidence of any other metastases: a case report. Br J Oral Maxillofac Surg 46: 499-501.

4. Reyes Court D, Encina S, Levy I (2007) Prostatic adenocarcinoma with mandibular metastatic lesion: case report. Med Oral Patol Oral Cir Bucal 12 E424-E427.

5. Van der Waal RI, Buter J, van der Waal I (2003) Oral metastasis from a prostate adenocarcinoma: report of a 24 cases. Br J Oral Maxillofac Surg 4: 3-6.

6. Hirshberg A, Leibovich P, Buchner A (1994) Metastatic tumors to the jawbones: analysis of 390 cases. J Oral Pathol Med 23: 337-341.

7. Boniello R, Gasparini G, D’Amato G, Di Petrillo A, Pelo S (2008) TMJ metastasis: A unusual case report. Head and Face Med 4: 8.

8. Pruckmayer M, Glaser C, Nasel C, Lang S, Rasse M, et al. (1996) Bone metastasis with superimposed osteomyelitis in prostate cancer. J Nucl Med 37: 999-1001.

9. Zhang FG, Hua CG, Shen ML, Tang XF (2011) Primary tumor prevalence has an impact on the constituent ratio of metastases to the jaw but not on metastatic sites. Int J Oral Sci 3: 141-152.

10. Wolujewicz MA (1980) Condylar metastasis from carcinoma of the prostate gland. Br J Oral Surg 18: 175-182.

11. Sastre J, Naval L, Muñoz M, Gamallo C, Diaz FJ (2005) Metastatic renal cell carcinome to the mandible. Otolaryngol Head Neck Surg 132: 663-664.

12. D'Silva NJ, Summerlin DJ, Cordell KG, Abdelsayed RA, Tomich CE, et al (2006) Metastatic tumors in the jaws. A retrospective study of 114 cases. JADA 137: 1667-1672.

13. Nagle RB, Cress AE (2011) Cress Metastasis Update: Human Prostate Carcinoma Invasion via Tubulogenesis Prostate Cancer 249290.

14. Reyes Court D, Encina S, Levy I (2007) Prostatic adenocarcinoma with mandibular metastatic lesion: case report. Med Oral Patol Oral Cir Bucal 12 : E424-E427. 